\title{
MORBIMORTALIDADE DA RECONSTRUÇÃO DE TRANSITO INTESTINAL COLÔNICA EM HOSPITAL UNIVERSITÁRIO - ANÁLISE DE 42 CASOS
}

\author{
LUIZ CARLOS VON BAHTEN ${ }^{1}$, JOÃO EDUARDO LEAL NICOLUZZI ${ }^{1}$, FÁBIO SILVEIRA ${ }^{1}$, GUILHERME \\ MATIOLLI NICOLLELLI ${ }^{1}$, LILLIAN YURI KUMAGAI ${ }^{1}$, VANESSA ZENI DE LIMA ${ }^{1}$ \\ ${ }^{1}$ Serviço de Cirurgia Geral - Hospital Universitário Cajuru - Curitiba PR - PUCPR. Trabalho apresentado no XXVI \\ Congresso Brasileiro de Cirurgiões, 2005
}

\begin{abstract}
VON BAHTEN LC, NICOLUZZI JEL, SILVEIRA F, NICOLLELLI GM, KUMAGAI LY, LIMA VZ. Morbimortalidade da Reconstrução de Transito Intestinal Colônica em Hospital Universitário - Análise de 42 Casos. Rev bras Coloproct, 2006;26(2):123-127.

RESUMO: OBJETIVOS - Analisar as características demográficas, a mortalidade e morbidade associada ao procedimento. MÉTODOS - Estudo retrospectivo dos casos de reconstrução intestinal colônica um hospital universitário. Todos os pacientes tiveram o cólon preparado por solução de manitol. RESULTADOS - Do total de 42 pacientes, 80,9\% (n=34) eram do sexo masculino com idade média de 42 anos. Causas que levaram a confecção da ostomia: $50 \%$ traumáticas, 29\% abdome agudo clínico. A colostomia terminal foi o tipo de ostomia preferencialmente realizada em $65 \%$ dos casos acompanhado pela colostomia em alça com $35 \%$ dos casos. A técnica empregada para a anastomose foi predominantemente a manual, realizada em 69,05\% dos casos (n=29). 0 tempo médio de internação hospitalar foi de 8,74 dias. $O$ índice de morbidade global foi de $26,2 \%$ (n=11), destacando-se a reoperação em 9,52\% (n=4) e a fístula em 7,14\% (n=3) dos casos. Não ocorreu infecção de ferida operatória nessa série. A mortalidade foi de $2,38 \%$ $(n=1)$. CONCLUSÕES - Os resultados obtidos em um hospital universitário são semelhantes aos relatados na literatura mundial. Cuidados pré e pós operatórios adequados se somam a experiência do cirurgião nas cirurgias de reconstrução de trânsito. A escolha da técnica cirúrgica deve ser padronizada através de trabalho randomizado, permitindo adoção de protocolo.
\end{abstract}

Descritores: colostomia, fechamento colostomias, morbidade.

\section{INTRODUÇÃO}

Desde a primeira descrição da realização de colostomia pelo francês Littré ${ }^{1}$, a utilização de estomas e suas indicações foram modificadas conforme a evolução da medicina. Sua utilização como auxílio terapêutico das afecções colorretais é bem definido, porém a carga de morbi-mortalidade associada à reconstrução do trânsito intestinal é ainda motivo de preocupações.
As particularidades técnicas das cirurgias de reconstrução de trânsito, desde o preparo préoperatório, meios de reconstrução e cuidados pósoperatórios são partes integrantes e importantes nos currículos das residências médicas em cirurgia geral. Relata-se na literatura taxas de morbidade variando de $0-50 \%$ e de mortalidade de $0-4,5 \%^{2,3}$ associadas a esse procedimento cirúrgico, dessa forma o conhecimento dos resultados obtidos permite o conhecimento dos fatores relacionados a esses índices e sua análise a melhora do serviço prestado.

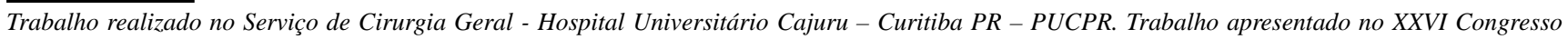
Brasileiro de Cirurgiões, 2005

Recebido em 21/03/2006

Aceito para publicação em 31/05/2006 


\section{OBJETIVOS}

Analisar as características demográficas, a mortalidade e morbidade associada ao procedimento em um ambiente de hospital universitário.

\section{PACIENTES E MÈTODOS}

Análise retrospectiva dos prontuários dos pacientes submetidos à cirurgia de reconstrução do trânsito intestinal colônico em um período de dois anos (2002-04) em um ambiente de hospital universitário. Todos os pacientes submetidos à reconstrução de trânsito tiveram suas ostomias confeccionadas no serviço, com programação de reconstrução via ambulatorial. O protocolo foi preenchido procurandose avaliar os dados demográficos, a causa indicadora da ostomia, o tempo de permanência da mesma, tipo de ostomia realizado, fatores relacionados ao procedimento cirúrgico per si, período de internação e fatores causadores de morbi-mortalidade.

O protocolo de preparo pré-operatório envolve solução de manitol a $20 \%(500 \mathrm{ml})+500 \mathrm{ml}$ de líquidos sem resíduos 12 horas antes da cirurgia, seguida de mais 1 litro de água, sendo os 2 litros ingeridos em um período de 2 horas. Nos casos indicados (colostomia terminal) uma lavagem com solução de glicerina via retal era realizado concomitantemente. Todos os paciente eram submetidos a exame contrastado (enema opaco) antes das intervenções cirúrgicas.

Antibioticoprofilaxia utilizada consiste em gentamicina (240mg EV) e metronidazol (500mg EV) na indução anestésica mantidas por um período de 24 horas.

\section{RESULTADOS}

Foram estudados 42 prontuários de pacientes submetidos à reconstrução de trânsito intestinal. Desses 42 pacientes, $34(80,95 \%)$ eram do sexo masculino (Tabela-1). Idade média de 42,04 anos. Dentre as causas que levaram a confecção da colostomia destacam-se as causas traumáticas em $45,23 \%$ dos casos, seguida das causas clínicas em $35,72 \%$ dos casos (Tabela-2). As causas de ostomia denominadas como "outras" incluem fístulas complexas, síndrome de Fournier e fístulas reto-vaginais. Os ferimentos por arma de fogo predominam nas causas traumáticas em $23,8 \%$ dos casos.

O tipo de colostomia mais frequentemente realizada foi a terminal em $64,3 \%(n=27)$ dos casos, seguida da colostomia em alça nos restantes 35,7\% $(n=15)$. Não foram observadas complicações (prolapso, hérnia para-estomal) relacionadas à ostomia no período de pré-fechamento. O tempo médio de permanência com a ostomia antes do fechamento foi de 6,53 meses.

O tipo de anestesia predominantemente utilizada foi a geral em 90,47\% casos (Gráfico-1).

Em relação à técnica cirúrgica empregada para a anastomose houve predomínio da sutura manual em 69,05\% dos casos, utilizando-se anastomose mecânica (endoluminal) nos restantes $30,95 \%$. Dentre os pacientes submetidos à anastomose manual, a realizada em 2 planos predominou em 68,97\% dos casos, cabendo aos restantes $31,03 \%$ dos casos a sutura em plano único. A drenagem da cavidade não foi realizada em $61,91 \%$ dos casos.

O índice de morbidade global em nossa série foi de $26,18 \%$, com predomínio das reoperações em $9,52 \%$ e fístula anastomótica em $7,14 \%$ dos casos (Tabela-3).

Tabela 1 - Distribuição quanto ao sexo.

\begin{tabular}{lll}
\hline & Número Casos & $\boldsymbol{\%}$ \\
\hline Masculino & 34 & 80,95 \\
Feminino & 8 & 19,05 \\
& 42 & 100 \\
\hline
\end{tabular}

Tabela 2 - Causas das indicações de ostomias.

\begin{tabular}{lcl}
\hline & No Casos / \% & Total \\
\hline Trauma & 19 & $45,23 \%$ \\
Ferida arma branca (FAB) & $1 / 2,38$ & \\
Ferida arma de fogo (FAF) & $10 / 23,8$ & \\
Trauma fechado & $8 / 19,05$ & \\
Clínico & 15 & $35,72 \%$ \\
Obstrutivo & $7 / 16,67$ & \\
Inflamatório & $8 / 19,05$ & \\
Outros & $8 / 19,05$ & $19,05 \%$ \\
& $42 / 100$ & $100 \%$ \\
\hline
\end{tabular}




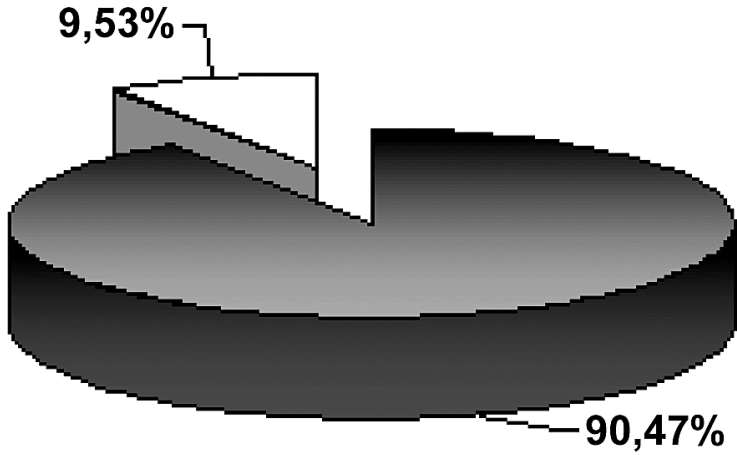

geral

Gráfico 1

Não observamos infecção de ferida operatória e deiscência de sutura abdominal em nossa série.

Houve um caso de óbito em um paciente de 76 anos que no pós-operatório apresentou fístula da anastomose, drenado, que após a reoperação evolui com sepse sem sucesso no tratamento.

Dos quatro pacientes reoperados, três haviam sido drenados, sendo que apenas um assim não o havia. Dos 3 casos de fístula, uma ocorreu em caso de sutura em 2 planos e duas em sutura mecânica. Todas as

Tabela 3 - Índice de morbidade.

\begin{tabular}{lcc}
\hline & No Complicações & \% \\
\hline Fístula & 3 & 7,14 \\
Pneumonia & 1 & 2,38 \\
Íleo prolongado & 1 & 2,38 \\
Reoperação & 4 & 9,52 \\
Óbito & 1 & 2,38 \\
Infecção urinária & 1 & 2,38 \\
TOTAL & 11 & 26,18 \\
\hline
\end{tabular}

complicações ocorreram em pacientes submetidos à reconstrução de trânsito após cirurgia de Hartmann.

\section{DISCUSSÃO}

A reconstrução do trânsito intestinal é considerada uma cirurgia de execução difícil ${ }^{(4,5)}$, com vários fatores e detalhes técnicos a serem observados ${ }^{(6)}$. Seus não desprezíveis índices de morbi-mortalidade relatados na literatura corroboram com o consenso acerca da dificuldade de sua realização (Tabela-4).

Vários fatores de risco tem sido implicados na literatura no desenvolvimento de complicações após o fechamento de colostomia, por exemplo, experiência do cirurgião ${ }^{(5,11)}$, doença primária ${ }^{(2)}$, localização do estoma $^{(12)}$, técnica operatória ${ }^{(13)}$ e o tempo de intervalo entre a primeira operação e o seu fechamento ${ }^{(3,11,14-16)}$. Infelizmente os resultados são conflitantes entre as séries relatadas.

Em nosso estudo, assim como em outros ${ }^{(17)}$, encontramos uma predominância do sexo masculino e

\section{Tabela 4}

\begin{tabular}{lccc}
\hline & No pacientes & Morbidade & Mortalidade \\
\hline Bocic et al $^{(7)}$ & 132 & $36,2 \%$ & $1,7 \%$ \\
Bannura et al $^{(8)}$ & 100 & 34 & 1,7 \\
Habr-Gamma et al $^{(9)}$ & 73 & 34,2 & 3,6 \\
Carreiro et al $^{(10)}$ & 35 & $25,7 \%$ & 0 \\
Curi et al $^{(6)}$ & 67 & 19,2 & 0 \\
\hline
\end{tabular}


tendo a violência como a principal indutora da confecção de colostomias, o que reflete a realidade social e a vocação de nosso hospital para o atendimento de emergências.

As ostomias além de desagradáveis para o paciente $^{(18)}$, trazem uma série de complicações pela sua presença, como infecção de parede, prolapso, oclusão intestinal e hérnias para-estomais, variando sua incidência de $10-60 \%{ }^{(19,20)}$. A ausência dessas complicações em nossa série não corrobora com alguns dados da literatura ${ }^{(21)}$ que demonstram um maior índice de complicações nos pacientes operados por cirurgiões gerais, visto que todos os pacientes de nossa série foram operados em um âmbito de residência médica em cirurgia geral.

Outros fatores considerados importantes na diminuição de complicações pós-operatórias, como a antibioticoprofilaxia e preparo mecânico do cólon ${ }^{(22)}$ são realizados de maneira rotineira e uniforme em nosso serviço.

O período médio de fechamento de uma colostomia aceito pela comunidade médica varia de 12 a 16 semanas, pelo fato que ocorreriam maiores índices de complicações se o fechamento ocorresse em um período inferior a 3 meses $^{(3,23)}$. Esses dados são questionados por alguns centros, que preconizam uma reconstrução mais precoce sem um aumento significativo nas taxas de complicações ${ }^{(10,11,22,25)}$. Os trabalhos ainda não demonstram claramente uma diferença significativa nos dois tipos de abordagem, levando ainda em consideração a diferença de reconstrução de uma colostomia terminal ou em alça em relação ás complicações ${ }^{(26,27)}$. Nosso serviço adota a prática de aguardar 90 dias para o fechamento de uma ostomia, nossos números de 6,53 meses para o fechamento reflete a sobrecarga do sistema de saúde o que atrasa a reoperação desses pacientes, talvez refletindo em um aumento da morbidade.
Quando consideramos a técnica empregada para a reconstrução do trânsito observamos que nosso serviço ainda não possui um consenso na realização das anastomoses manuais em um ou dois planos. Observamos que os casos de fístula envolveram anastomoses mecânicas e uma realizada em dois planos, porém sem atingir nível de significância estatística. As anastomoses em 2 planos são consideradas pela literatura mais propensas a desenvolverem estenoses $^{(28,29)} \mathrm{e}$ as suturas mecânicas são consideradas seguras e propensas a complicações em taxas semelhantes às suturas manuais ${ }^{(30)}$.

As complicações mais comuns da cirurgia são as infecciosas e a fístulas ${ }^{(4-6,31,32)}$, nosso índice de complicações de $26,18 \%$ está de acordo com o verificado na literatura.

A utilização de drenagem, questão de eternas controvérsias entre especialidades e cirurgiões, não foi capaz de prevenir reoperações em nossa série, visto que 3 dos 4 pacientes reoperados foram drenados. Todos os pacientes que fistulizaram estavam drenados e todos necessitaram reoperação.

\section{CONCLUSÕES}

A reconstrução de trânsito intestinal continua sendo uma cirurgia com índices elevados de morbidade. Porém, com adequada orientação, preparo pré-operatório e respeito aos princípios cirúrgicos adequados e consistentes resultados podem ser obtidos em um ambiente de hospital universitário de cirurgia geral. Isso leva a crer que outros fatores além da experiência do cirurgião e sua especialidade influenciam nesses índices.

Nossos resultados ainda nos obrigam a manter a tendência já crescente no serviço de se evitar a drenagem da cavidade abdominal e realizar as anastomoses manuais em plano único.

SUMMARY: OBJECTIVES - To study demographyc caractheristics, morbidity and mortality associated to the procedure. METHODS - Retrospective study of colostomy closure in 42 patients. Male sex was predominant $(80,9 \%)$ with median age of 42 years. Causes of colostomy were traumatic in $\mathbf{5 0 \%}$ and clinic acute abdomen in $29 \%$ of the cases. Terminal colostomy was the more frequent procedure $(65 \%)$ followed by loop colostomy in $35 \%$ of the cases. The predominant anastomotic technique was manual $(69,05 \%)$. The morbidity rate was $26,2 \%$, including reoperation $(9,52 \%)$ and colonic fistulae in $7,14 \%$ cases. No postoperative wound infection was observed. Overall mortality rate was $2,38 \%$. CONCLUSION - The results observed in a university hospital are as good as reported in world literature. Adequate pre and postoperative care works together with surgeon experience on colostomy closure surgeries. The surgical technique must be adopted using a randomized study, allowing establishment of a protocol.

Key words: colostomy, colostomy closure, morbidity. 


\section{REFERÊNCIAS BIBLIOGRÁFICAS}

1. Kretschmer, P. Estomas Intestinais. 1a.ed.,Rio de Janeiro, Interamericana, 1980.

2. Pittman DM, Smith LE. Complications of colostomy closure. Dis Colon Rectum 1985; 28: 836-43.

3. Knox AJ, Birkett FDH, Collins CD. Closure of colostomy. Br J Surg 1971;58:669-72.

4. Thal ER, Yeary EC. Morbidity of colostomy closure following colon trauma. J Trauma 1980; 20: 287-91.

5. Demetriades D, Pezikis A, Melissas J et al. Factors influencing the morbidity of colostomy closure. Am J Surg 1988; 155: $594-6$.

6. Curi A, Moreira Júnior H, Mascarenhas JCS, Moreira JPT, Almeida AC, Azevedo IF, Louza LR, Moreira H. Morbimortalidade associada à reconstrução de trânsito intestinal - Análise de 67 casos. Rev Bras Coloproct 2002; 22(2):88-97.

7. Bocic GA, Jensen CB, Abedrapo MM, Garrido RC, Pérez GO, Cúeneo AZ. Colostomías e Ileostomías: 8 Años de experiencia clínica. Rev Hosp Clin Univ Chile 1999; 10(3):195-200.

8. Bannura GC, Perales CG, Contreras JP, Valencia CE, Melo CL. Reconstituición del tránsito intestinal luego de la operación de Hartmann: análisis de 100 pacientes. Rev Chil Cir 1999; 51(4):359-66.

9. Habr-Gama A, Teixeira MG, Vieira MJF, Miléu LF, Laurino Neto R, Pinotti HW. Operação de Hartmann e suas consequiências. Rev Bras Colo-proctologia 1997; 17(1):5-10.

10. Carreiro PRL, Lázaro da Silva A, Abrantes WL. Fechamento precoce das colostomias em pacientes com trauma do reto: Um estudo prospectivo e casualizado. Rev. Coleg. Bras. Cir 2000;27 (5):298-304.

11. Aston, C.M., Everett, W.G.: Comparison of early and late closure of transverse loop colostomies. Ann. R. Coll. Surg. Engl. 66:331, 1984

12. Anderson, E., Carey, L.C., Cooperman, M.: Colostomy closure-a simple procedure? Dis. Colon Rectum 22:466, 1979

13. Kohler, A., Athanasiadis, S., Nafe, M.Postoperative results of colostomy and ileostomy closure: a retrospective analysis of three different closure techniques in 182 patients. Chirurg 65:529, 1994.

14. Salley, R.K., Bucher, R.M., Rodning, C.B.: Colostomy closure: morbidity reduction employing a semi-standardized protocol. Dis. ColonRectum 26:319, 1983.

15. Parks, S.E., Hastings, P.R.: Complications of colostomy closure.Am. J. Surg. 149:672, 1985

16. Freund, H.R., Raniel, J., Muggia Sulam, M.: Factors affecting the morbidity of colostomy closure: a retrospective study. Dis. Colon Rectum 25:712, 1982.
17. Burch JM, Feliciano DV, Mattox KL. Colostomy and drainage for civilian rectal injuries: is that all? Ann Surg 1989;209; 600-11.

18. Velmahos GC, Degiannis E, Wells M et al. Early closure of colostomies in trauma patients - a prospective randomized trial. Surg 1995; 118: 815-20.

19. Abcarian H, Pearl RK. Estomas. (1a ed.),Clínicas da América do Norte. Rio de Janeiro, Interlivros, 1988; pp. 1365-1376.

20. Pearl RK, Prasad ML, Orsay CP. et al: Early local complications from intestinal stomas. Arch Surg 1985; 120:1145-1147.

21. Park JJ , Del Pino A, Orsay CP, Nelson RL, Pearl RK, Cintron JR, Abcarian H. Stomas complications: The Cook county hospital experience. Dis Colon Rectum. 1999;42:1575-1580.

22. Riesener KP, Lehnen W, Hofer M, Kasperk R, Braun JC, Schumpelick V. Morbidity of ileostomy and colostomy closure: impact of surgical technique and perioperativa treatment. World J Surg 21, 103-108, 1997.

23. Yakimets WW: Complications of closure of loop colostomy. Can J Surg 1975; 18:366-70.

24. Lewis A, Weeden D. Early closure of transverse loop colostomies.Annals R Coll Surg Engl 1982; 64: 57-8.

25. Geoghegan JG, Rosemberg IL. Experience with early anastomosis after the Hartmann procedure. Ann R Coll Surg Engl 1991; 73: 80-2.

26. Parks SE, Hastings PR. Complications of colostomy closure.Am J Surg 1985; 149: 672-5.

27. Varnell J, Pemberton LB. Risk factors in colostomy closure.Surg 1981; 89: 683-6.

28. Dunn DH, Decanini CO, Delaney JP: Colonic anastomosis: inverted and everted, sutured and stapled. Surg Forum 1980; 31:159-161.

29. Ravitch MM, Brolin R, Kolter J, et al: Studies in the healing of intestinal anastomosis. World J Surg 1981; 5:627-637.

30. Barnett JE, Pheils M: Closure of colostomy. Aust. N Z J Surg 1976;46: 131-3.

31. Goligher JC, Morres C, McAdam WAF, et al: A controlled trial of inverting versus everting intestinal suture in clinical large bowel surgery. Br J Surg. 1970; 57:817-822.

32. Adan YG, Volk H, State D: Low colorectal anastomosis after resection for cancer. Surg Gynecol obstet 1967;125;1259-1264

Endereço para correspondência: LUIZ CARLOS VON BAHTEN Rua Martin Afonso 2642, ap1902 80530-030 Curitiba (PR) vonbahten@yahoo.com.br 